\title{
Prevalence of Impacted Lower Canines and Lower Second Premolars among Dental Students
}

\section{Emad Hammody Abdulla*, Ali Abd Al Kareem Mohammed, Shafaa Abd Al Kadum and Reyad Jafar}

College of Dentistry, Ibn Sina University of Medical and Pharmaceutical Sciences,

Baghdad, Iraq

*Corresponding Author: Emad Hammody Abdulla, Assistant Professor, College of Dentistry, Ibn Sina University of Medical and Pharmaceutical Sciences, Baghdad, Iraq.
Received: July 26, 2021

Published: September 07, 2021

(C) All rights are reserved by Emad Hammody Abdulla., et al.

\begin{abstract}
Introduction: Mandibular second premolar came in the third grade after third permanent molars and maxillary permanent canines in frequency of impaction. They are found impacted at different angulation and positions and should be carefully evaluated radio graphically during treatment planning. The incidence of impacted mandibular canines in the mandible is low as that in the maxilla, few sound studies based on large patient samples found.

Aim of this Study: The aim of this study was to evaluate the prevalence of impacted lower canines and lower premolars among Iraqi students in the dental colleges.

Materials and Methods: This study involved 147 dental students, and these cases had presented to college of dentistry in Ibn sina University for medical and pharmacological sciences, Iraq. Duration of the study from February 2021 to June 2021.

Results: Total case examined 146, 24 case excluded because of age range. The Results of 122 cases showed a total of 7 impacted mandibular canine teeth were found in 7 of the 122 patients (5.73\%) with 5 being in females (ages 18 - 25) and 2 in males (ages 18 25). In two patients Impacted lower canines were bilateral and the other cases were unilateral. A total of 5 impacted lower premolars were found in 5 of 122 patients (4.09\%) with 3 being in females (age 18 - 25) and 2 being in males (age 18 - 25), this impaction was bilateral in 1 case and unilateral in the other one.

Conclusion: Within the limitations of this study, the following was drawn: The prevalence of impacted lower canines and lower second premolars among age group 18 - 25 is 4 - 8\%. The increase in accidental findings of impacted mandibular canine and second premolars should encourage the community to raise awareness and educate the population about the clinical implications and the importance of implementing preventive and interceptive procedures.
\end{abstract}

Keywords: Impacted Lower Canines; Lower Second Premolars; Dental Students

\section{Introduction}

Impaction may be defined as the failure of a tooth to erupt, usually caused by crowding or an obstruction. A failure of tooth to erupt due to in the eruptive mechanism (primary failure of eruption) is rare. Some medical conditions (e.g. cleidocranial dysplasia) can be associated with multiple impactions [1].
The etiology of impaction of teeth other than the third molars is not well defined. Intrusive trauma to the periodontal ligaments which are extensively damaged may cause impaction that is common in the anterior segment. Adjacent tooth, dense bone or soft tissue may obstructed the eruption of a tooth and may cause impaction. The early loss of deciduous $2^{\text {nd }}$ molars without managed by 
placing of space maintainer, tooth/arch size discrepancy may cause crowding in the buccal segment, this leads to insufficient space for the $2^{\text {nd }}$ premolar to erupt, so the premolar may either erupt ectopically or become impacted. Periodontal disease, dental caries, odontogenic cyst and tumors, pain of unexplained origin, jaw fracture and resorption of the root of the adjacent tooth Impacted teeth are possible factors in the etiology of partially or completely impacted teeth [2]. Genetic and environmental factors may affect the multifactorial nature of tooth eruption, at any stage of tooth development [3]. Impaction of tooth is a common problem in clinical orthodontics. It is considered when the tooth is an intraosseous position and no clinical signs of its eruption after expected time, when root of impacted teeth is fully formed with at least after six months of contralateral tooth eruption and one year after expected chronological age [4]. Most impaction remain asymptomatic but, risk remains of follicular enlargement, root resorption of adjacent teeth and infection [5]. The early detection of impacted canine is of greatimportance.

Impacted canines may worse dental health and could cause resorption roots of neighboring teeth, their strategic position at the angle of arch is significant in maintenance of the harmony and symmetry of occlusal relationship, in determining the contours of the mouth [6]. There are many reasons for canines impaction, an ectopic eruption, inadequate space, supernumerary teeth, premature loss of the deciduous canine, excessive crown length, hereditary factors, a suspected pathological condition, infection, functional disturbances of the endocrine glands, tumors, cysts and trauma.

Impacted mandibular canines are located on the labial aspect of the dental arch also more likely to be than that in impacted maxillary canines. The mandibular canines are affected by pathological condition in a lower ratio than that in third molars and premolars [7].

Mandibular second premolars impaction: Mandibular second premolars camein the third grade third after third permanent molars and maxillary permanent canines in frequency of impaction. The mandibular second premolar is highly variable developmentally. Among the reported developmental anomalies are agenesis, abnormal tooth germ position, pathology such as inflammatory or dentigerous cyst, an ankylosed primary molar, presence of supernumerary teeth, odontomas and distal inclination of the developing tooth. Local factors like mesial drift of teeth arising from the premature loss of the primary molars; ectopic positioning of the developing premolar tooth buds; or the second most frequently impacted tooth was found to be the mandibular second premolar, excluding third molars. Some systemic factors involved cleidocranial dysplasia, osteoporosis, hypothyroidism, hypopituitarism and Down's syndrome. The mandibular premolars erupt after the mandibular first molar and mandibular canines so if the space for eruption of premolars is inadequate, one of them usually the second premolar remains unerupted and chances of being impacted are high. The prevalence for mandibular premolars impaction in adults has the range be $0.2 \%$ to $0.3 \%$ [8].

\section{Aim of the Study}

The aim of this study was to evaluate the prevalence of impaction of lower canine and lower second premolars, among Iraqi Students in the dental colleges, of Ibn sina University for medical and pharmacological sciences, Iraq, Baghdad.

\section{Materials and Methods}

This study involved 147 students, only 122 from them for age 18 - 25 years old only for dental students and these cases had presented to college of dentistry in Ibn sina University for medical and pharmacological sciences, Iraq. Study started from February ended in June 2021. The 25 cases were excluded because of the age of students. Tooth was considered impacted when it was not aligned with the rest of the teeth in either of the lower dental arch. Data regarding age, sex, stage of studying, number of impacted teeth, arch involved, and type of impaction were obtained from patient's clinical examination and recorded in special case sheet prepared for this study, the examine done on Dental chair, using disposable mirror. The prevalence of impacted lower canine and premolars in relation to age and gender, was assessed by incidence and percentage.

\section{Results}

Total case examined 146, 24 case excluded because of age range. The results of 122 cases showed a total of 7 impacted mandibular canine teeth were found in 7 of the 122 patients (5.73\%) with 5 being in females (ages 18 - 25) and 2 in males (ages 18 - 25). In two patients impacted lower canines were bilateral and the other cases were unilateral. A total of 5 impacted lower premolars were found in 5 of 122 patients (4.09\%) with 3 being in females (age 18 - 25) and 2 being in males (age 18 - 25), this impaction was bilateral in 1 case and unilateral in the other (Table 1-3). 


\begin{tabular}{|l|c|c|c|c|c|c|c|}
\hline Type of tooth impaction & Total cases & Incidence & Bilaterally & Males & Females & Mean age & Age range years \\
\hline Impacted lower canine & 122 & 7 & 2 & 52 & 70 & 21.5 & $18-25$ \\
\hline Impacted lower premolar & 122 & 5 & 1 & 52 & 70 & 21.5 & $18-25$ \\
\hline
\end{tabular}

Table 1: Incidence gender, age ration for impacted lower canine, lower premolar.

\begin{tabular}{|l|c|c|}
\hline Gender & $\begin{array}{c}\text { Impacted } \\
\text { lower canine }\end{array}$ & $\begin{array}{c}\text { Impacted lower } \\
\text { premolar }\end{array}$ \\
\hline Males & 2 & 2 \\
\hline Females & 5 & 3 \\
\hline Total & 7 & 5 \\
\hline
\end{tabular}

Table 2: Incidence gender ration for impacted lower canine, lower premolar.

\begin{tabular}{|l|l|l|l|}
\hline Type of tooth impaction & $\begin{array}{l}\text { Total } \\
\text { cases }\end{array}$ & Incidence & $\begin{array}{l}\text { Prevalence } \\
\text { \% }\end{array}$ \\
\hline Impacted lower canine & 122 & 7 & 5.7 \\
\hline Impacted lower premolar & 122 & 5 & 4.1 \\
\hline
\end{tabular}

Table 3: Prevalence of teeth impaction in the study.

\section{Discussion}

In our study teeth were found that prevalence of impacted mandibular canine (5.73\%) with (ages 18 - 25) and we found prevalence of impacted lower premolars of (4.09\%) with in (age 18 - 25), numbers of studies demonstrated the prevalence of impacted permanent canine, in the maxilla it reported between $0.8 \%$ and $5.4 \%$. Canine impaction is relatively common and has been reported extensively in different populations, ranging from 0.8 - 3.6\% [4].

The impaction of the canine had the greatest occurrence, $28.10 \%$ of all impacted teeth [9]. Results in the mandible less percentage had been reported $0.3 \%$ and $0.44 \%$. The impacted canine in the Sudanese sample were similar to the finding by Ericson and Kurol among Swedish sample (2.05\%). However, it was less than the finding among Saudi sample (3.6\%) and Turkish (3.73\%) [10]. In studies prevalence of impacted premolars has been reported to be $0.5 \%$, with a range of $0.1 \%$ to $0.3 \%$ for maxillary premolars and $0.2 \%$ to $0.3 \%$ for mandibular premolars [11].

The prevalence of impacted premolars in one Iraqi study has been reported as be for maxillary premolars (8.91\%), while for mandibular premolars (91.08\%). The prevalence of impacted

maxillary first and second premolars (2.97\%) and (5.94\%,) respectively and the prevalence of impacted mandibular first and second premolars was (11.88\%) and (79.20\%), respectively [13]. Our study has some limitations, the sample size was small and the sample population was only representative of the patient pool at the Faculty of Dentistry. Wider population groups should be studied in our country. However, some believe that the prevalence rates of teeth impaction may reflect the prevalence rates of these dental anomalies and help in the future planning for general health care.

\section{Conclusion}

Within the limitations of this study, the following was drawn: The prevalence of impacted lower canines and lower second premolars among age group 18 - 25 is 4 - 8\%. [impacted mandibular canine teeth were found in prevalence of (5.73\%) with (ages 18 - 25) impacted lower premolars were found in prevalence of (4.09\%) with in (age 18 - 25)]. The increase in accidental findings of impacted mandibular canine and second premolars should encourage the community to raise awareness and educate the population about the clinical implications and the importance of implementing preventive and interceptive procedures.

\section{Bibliography}

1. Daljit Gill. "Orthodontics at a Glance, Laboratory of Congress Cataloging-in-publication”. Data (2008): 68-71.

2. Al-Faleh W. "Completely impacted teeth in dentate and edentulous jaws”. Pakistan Oral and Dental Journal (2009): 255260.

3. BJ Moxham and BK Berkovitz. "Interactions between thyroxine, hydrocortisone and cyclophosphamide in their effects on the eruption of the mandibular incisors". Archives of Oral Biology (1993): 1083-1087.

4. Beste Kamiloglu and Umay Kelahmet. "Prevalence of impacted and transmigrated canine teeth in a Cypriote orthodontic population in the Northern Cyprus area". BMC Research Notes 7.346 (2014). 
5. Bishara SE. "Impacted maxillary canines: a review". American Journal of Orthodontics and Dentofacial Orthopedics (1992): 15-20.

6. Raja A Mustafa and Amal H Abuaffan. "Prevalence of impacted canines among Sudanese university students". Brazilian Dental Science 17.4 (2014): 27-33.

7. Simon Camilleri. "Transmigration of Mandibular Canines-A Review of the Literature and a Report of Five Cases". The Angle Orthodontist 73 (2003): 753-762.

8. Kalia V and Angela M. "Mandibular premolar impaction Scholarly Research Exchange (2009).

9. Hou R and Kong LAJ. "Investigation of impacted permanent teeth except the third molar in Chinese patient through an Xray study". Journal of Oral and Maxillofacial Surgery (2010): 762-767.

10. A B Mustafa. "Prevalence of Impacted Pre-Molar Teeth in College of Dentistry, King Khalid University, Abha, Kingdom of Saudi Arabia". Journal of International Oral Health 7.6 (2015): 1-3.

11. Sağlam AA and Tüzüm MS. "Clinical and radiologic investigation of the incidence, complications, and suitable removal times for fully impacted teeth in the Turkish population". Quintessence International 34 (2003): 53-59.

12. Oikarinen VJ and Julku M. "Impacted premolars. An analysis of 10000 orthopantomograms". Proceedings of the Finnish Dental Society 70 (1974): 95-98.

13. Zainab $\mathrm{H}$ and Al-Ghurabi. "Prevalence of impacted premolar in Iraqi sample". IOSR Journal of Dental and Medical Sciences 13.6 (2014): 16-20.

\section{Volume 5 Issue 10 October 2021}

C All rights are reserved by Emad Hammody Abdulla., et al. 